\title{
Expression of cancer-associated fibroblast markers in advanced colorectal cancer
}

\author{
RIE NISHISHITA $^{1,2}$, SATOKO MOROHASHI ${ }^{1}$, HIROKO SEINO $^{1}$, YUNYAN WU ${ }^{1}$, TADASHI YOSHIZAWA ${ }^{1}$, \\ TOSHIHIRO HAGA ${ }^{1}$, KENSUKE SAITO $^{1}$, KENICHI HAKAMADA $^{3}$, SHINSAKU FUKUDA $^{2}$ and HIROSHI KIJIMA ${ }^{1}$ \\ Departments of ${ }^{1}$ Pathology and Bioscience, ${ }^{2}$ Gastroenterology and Hematology, \\ and ${ }^{3}$ Gastroenterological Surgery, Hirosaki University Graduate School of Medicine, Hirosaki, Aomori 036-8562, Japan
}

Received May 18, 2017; Accepted December 11, 2017

DOI: $10.3892 / \mathrm{ol} .2018 .8097$

\begin{abstract}
Colorectal cancer is one of the most common causes of mortality from cancer worldwide. Previous studies have demonstrated that cancer-associated fibroblasts (CAFs) promote neoangiogenesis and tumor growth for various tumors. The present study analyzed CAF markers, including $\alpha$-smooth muscle actin ( $\alpha$-SMA), collagen I, platelet-derived growth factor receptor- $\beta$ (PDGFR- $\beta$ ), and D2-40 (antibody recognizing podoplanin), and vessel markers, including cluster of differentiation (CD)31 and CD34, for 121 advanced colorectal cancer cases using a digital image analyzing technique. The association between CAF markers and vessel markers with clinicopathological factors was investigated. Furthermore, the association between CAF markers with each other, and their association with vessel markers was analyzed. Mean/median expression area of stromal and vessel markers in tumors were collagen I, 26.787\%; D2-40, 1.372\%; PDGFR- $\beta, 11.646 \% ; \alpha$-SMA-positive and desmin-negative myofibroblasts ( $\alpha$-SMA subtraction), $15.372 \%$; CD31, 3.635\%; and CD34, 2.226\%. The expression area of $\alpha$-SMA subtraction was significantly correlated with collagen I $(\mathrm{P}<0.001$, correlation rho=0.509). High levels of $\alpha-S M A$ subtraction $(\mathrm{P}=0.002)$, collagen I $(\mathrm{P}=0.040)$, and PDGFR- $\beta$ $(\mathrm{P}=0.040)$ expressions tended to be associated with high venous invasion. D2-40 did not correlate with other CAF and vessel markers. These results indicated that individual CAFs may have different expression patterns, and different strength effects for venous invasion in advanced colorectal cancer stroma.
\end{abstract}

\section{Introduction}

Cancer tissues are composed of cancer cells and the surrounding stroma including fibroblasts, vascular endothelial

Correspondence to: Dr Satoko Morohashi, Department of Pathology and Bioscience, Hirosaki University Graduate School of Medicine, 5 Zaifu-cho, Hirosaki, Aomori 036-8562, Japan

E-mail: msatoko@hirosaki-u.ac.jp

Abbreviation: CAF, cancer-associated fibroblast

Key words: colorectal cancer, cancer-associated fibroblast cells, and extracellular matrix. Recent studies have focused on the cancer-associated fibroblasts (CAFs), a major cellular component of the cancer stroma, and have demonstrated that CAFs promote neoplastic angiogenesis and tumor growth in various tumors (1-6).

Collagen I, D2-40 (antibody recognizing podoplanin), Platelet-derived grow th factor receptor- $\beta$ (PDGFR- $\beta$ ), and $\alpha$-smooth muscle actin ( $\alpha$-SMA) have been known as molecular/histopathological markers of CAFs (7). Podoplanin (D2-40) expressions in CAFs from various cancers have been studied (8). The majority of recently reports identified podoplanin (D2-40) expression of CAFs as an unfavorable marker of prognosis, such as lung cancer (9), breast cancer (10), and esophageal adenocarcinoma (11), while podoplanin expression of CAFs was shown as a favorable prognosis indicator of colorectal cancer $(12,13)$. Some previous studies have reported one of the above CAF markers, but there are no papers for analyzing the relationships among these CAFs using image technique in colorectal cancer. The relationships of CAF markers, such as collagen I, D2-40, PDGFR- $\beta$, and $\alpha$-SMA in advanced colorectal cancer are still unknown. We speculate synergic effects of individual CAF markers are important, and may significantly contribute neoplastic angiogenesis.

In this study, we analyzed histopathological expression of CAF markers in the human advanced colorectal cancers, as well as vessel markers (CD31 and CD34), because CAFs are thought to promote neoplastic angiogenesis in the cancer stroma. In addition, we examined the relations among the $\mathrm{CAF} /$ vessel markers and clinicopathological factors.

\section{Materials and methods}

Tissue specimens. A total 121 tumor samples from patients who underwent curative surgical resection for advanced colorectal cancer at the Hirosaki University Hospital between January 2008 and December 2009 were included. Informed consent was obtained from each patient regarding the use of clinical records and pathological specimens. Cancer had invaded the subserosa layer of the colorectal wall, and the clinical stages were stage IIA, IIIB, or IIIC according to the TNM classification of the UICC (14). Lymph nodes were evaluated histologically. No patient received preoperative chemotherapy, and no patient had metastasis of other 
organs. Ethical approval was obtained from the Hirosaki University Graduate School of Medicine Ethics Committee (Hirosaki, Japan).

Pathological analysis. For histopathological examination, all surgically resected specimens were fixed using $10 \%$ formalin, embedded in paraffin, and stained using hematoxylin and eosin. The histological features were assessed in the largest cross-sectional tumor section, and histological type, lymphatic invasion, venous invasion, and lymph node metastasis were evaluated according to the Japanese Classification of Colorectal Cancer (15). One hundred twenty-one cases of colorectal cancer were classified into well-differentiated tubular adenocarcinoma (tub1), moderately differentiated tubular adenocarcinoma (tub2), papillary adenocarcinoma (pap), poorly differentiated adenocarcinoma (por), and mucinous adenocarcinoma (muc). Papillary adenocarcinoma consists of papillary or villous architecture neoplastic glands. Histological type was classified into two groups: Differentiated type, tub1, tub2, or pap, and poorly differentiated type, por or muc. The degree of lymphatic invasion was classified as ly0, no invasion; ly1, minimal invasion; ly2, moderate invasion; or ly3, severe invasion. We regarded ly0 and ly1 as low-grade invasion and ly 2 and ly 3 as high-grade invasion. The degree of venous invasion was classified as the same as lymphatic invasion.

Immunohistochemical staining. For immunohistochemical examination, sections on microslides were deparaffinized using the standard avidin-biotin-peroxidase complex method with automated immunostainer (Benchmark XT; Ventana Medical System, Tucson, AZ, USA). The antibodies of clones and dilution ratios were $\alpha$-SMA (clone 1A4, dilution 1:100; Dako; Agilent Technologies, Inc., Santa Clara, CA, USA), desmin (clone D33, dilution 1:100; Dako; Agilent Technologies, Inc.), D2-40 (clone D2-40, cat. no. 413451, diluted antibody; Nichirei Biosciences, Inc., Tokyo, Japan), CD31 (clone JC70A, dilution 1:40; Dako; Agilent Technologies, Inc.), CD34 (clone QBEnd 10, DAKO, dilution 1:100), PDGFR- $\beta$ (clone C82A3, dilution 1:100; Cell Signaling Technology, Inc., Danvers, MA, USA), and collagen I (clone COL1A1, dilution 1:100; Rockland, Inc., Gilbertsville, PE, USA).

Image analysis. For the evaluation of each marker in the cancer stroma, we used an image analysis. We selected a hot spot of D2-40-positive stromal area for each case. When the D2-40 expression was not found, we captured an image of the site with the most CAFs. We used a microscope BX53 with an UPlanFL objection lens, $\mathrm{x} 4$ magnification, DP control software, and a DP-21 digital camera (Olympus, Tokyo, Japan) for captured images. ImageJ software (National Institutes of Health, Bethesda, MD, USA) was applied to analyze captured images. We captured immunohistochemical staining images, and adjusted phases in each case by using Adobe Photoshop software (Adobe ${ }^{\circledR}$ Photoshop ${ }^{\circledR}$ CC $2014{ }^{\circledR}$; Adobe Systems, Inc., San Jose, CA, USA) for image registration. We cropped out the maximum range that a cancer stroma could be evaluated from the adjusted image, and binarized them using the ImageJ software.

Evaluation of immunohistchemical staining expression area. We measured the percentage of immunostaining-positive lesions in the total cropped, binarized area for each immunostained slide. The binarized image shows immunostaining-positive and -negative lesions as black and white, respectively. For the examination of $\alpha$-SMA-positive myofibroblasts, we made an $\alpha$-SMA-desmin subtraction image using the subtraction mode of the ImageJ software (Fig. 1) because $\alpha$-SMA became positive for muscle tissues, such as muscularis mucosa and muscular layer, in addition to the myofibroblasts. The subtraction image shows the value of $\alpha$-SMA-positive and desmin-negative myofibroblasts in the cancer stroma. We called this subtraction image as $\alpha$-SMA subtraction. D2-40-, PDGFR- $\beta-$, and collagen I-positive lesions were made binarized and calculated expression area (the positive percentage of the cropped area) by using ImageJ software in the cancer stroma as CAF markers. CD31 and CD34 positive lesions were also made binarized and calculated as vessel markers by using Image J software.

Evaluation of immunohistchemical staining expression intensity. Three investigators (RN, SM, and HS) evaluated the immunohistochemical results in the captured images at low magnification (x40). There was unevenness in the level of PDGFR- $\beta$, D2-40, and collagen I staining intensity for each case, which required an evaluation by intensity score. The intensity of $\alpha$-SMA staining was strong and uniform for all cases, therefore, $\alpha$-SMA was evaluated by only expression area analysis. Immunohistochemical scores for PDGFR- $\beta$, collagen I, and D2-40 were evaluated according to the staining intensity as follows: Score 1, weak staining in stromal cell; score 2, moderate staining in stromal cell; score 3, severe staining in stromal cell (Fig. 2). The scores given by the three evaluators were summed for each case. We regarded cases with total score of 8 to 9 as high intensity and 3 to 7 as low intensity. The intensity of CD31/CD34 staining was strong and uniform for all cases, therefore, CD31/CD34 were evaluated by only expression area analysis.

Statistical analysis. The value of immunohistochemical expression area and intensity and pathological factor were compared by using Pearson's chi-square test or Fisher's exact test for categorical data. Normally distributed and homoscedastic data were analyzed by two-sample t-test, and non-normally distributed data were analyzed by the Wilcoxon rank sum test for continuous data. The median immunostaining expression area ( $\alpha$-SMA subtraction, CD31, and CD34) and each staining intensity score (collagen I, D2-40, and PDGFR- $\beta$ ) were compared by using Kruskal-Wallis test. $\mathrm{P}<0.05$ was considered to indicate a statistically significant difference. Each of the mean/median immunostaining expression percentage in the cancer stroma was compared using Spearman's rank correlation coefficient. Correlation was defined as statistically significant if the rho value (r) was $>0.4$. All statistical evaluations were performed using R (http://www.r-project.org) and EZR (Saitama Medical Center, Jichi Medical University, Saitama, Japan).

\section{Results}

Expression of CAF/vessel markers of colorectal cancer tissues. The clinicopathological characteristics of the 121 colorectal 

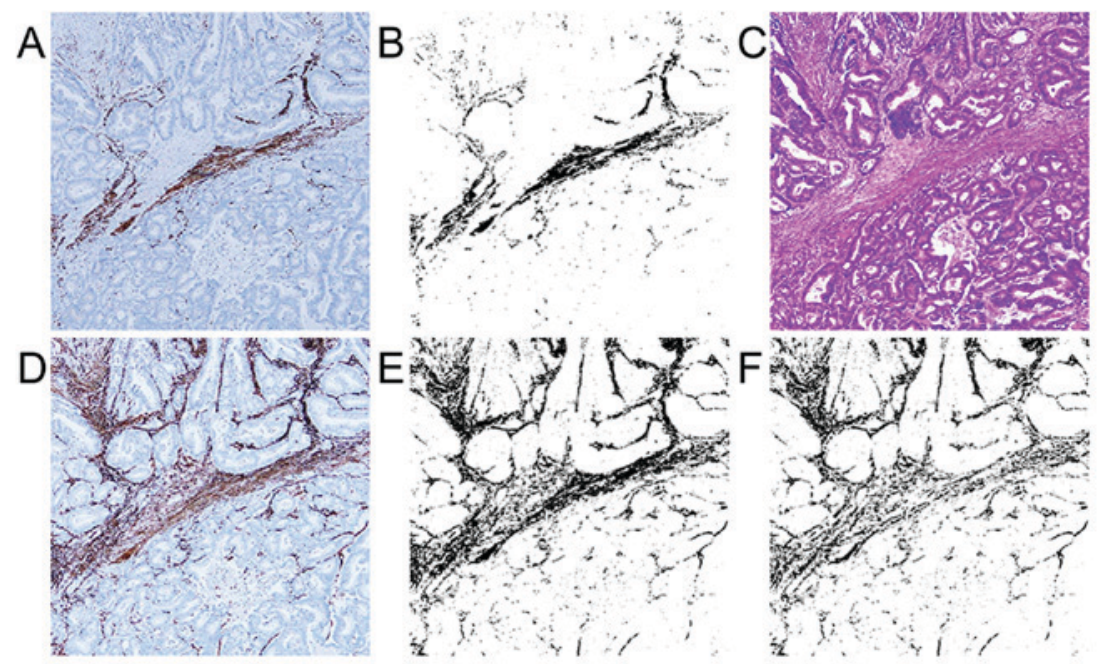

Figure 1. Imaging analysis for $\alpha$-smooth muscle actin ( $\alpha$-SMA) subtraction. (A) Immunohistochemical expression of desmin (magnification, x40); (B) binalization image of desmin (magnification, $\mathrm{x} 40$ ); (C) hematoxylin and eosin staining (magnification, $\mathrm{x} 40$ ); (D) immunohistochemical expression of $\alpha$-SMA (magnification, $\mathrm{x} 40$ ); (E) binalization image of $\alpha$-SMA (magnification, $\mathrm{x} 40$ ); and (F) image which obtained by subtracting desmin from $\alpha$-SMA ( $\alpha$-SMA subtraction) (magnification, $\mathrm{x} 40$ ).
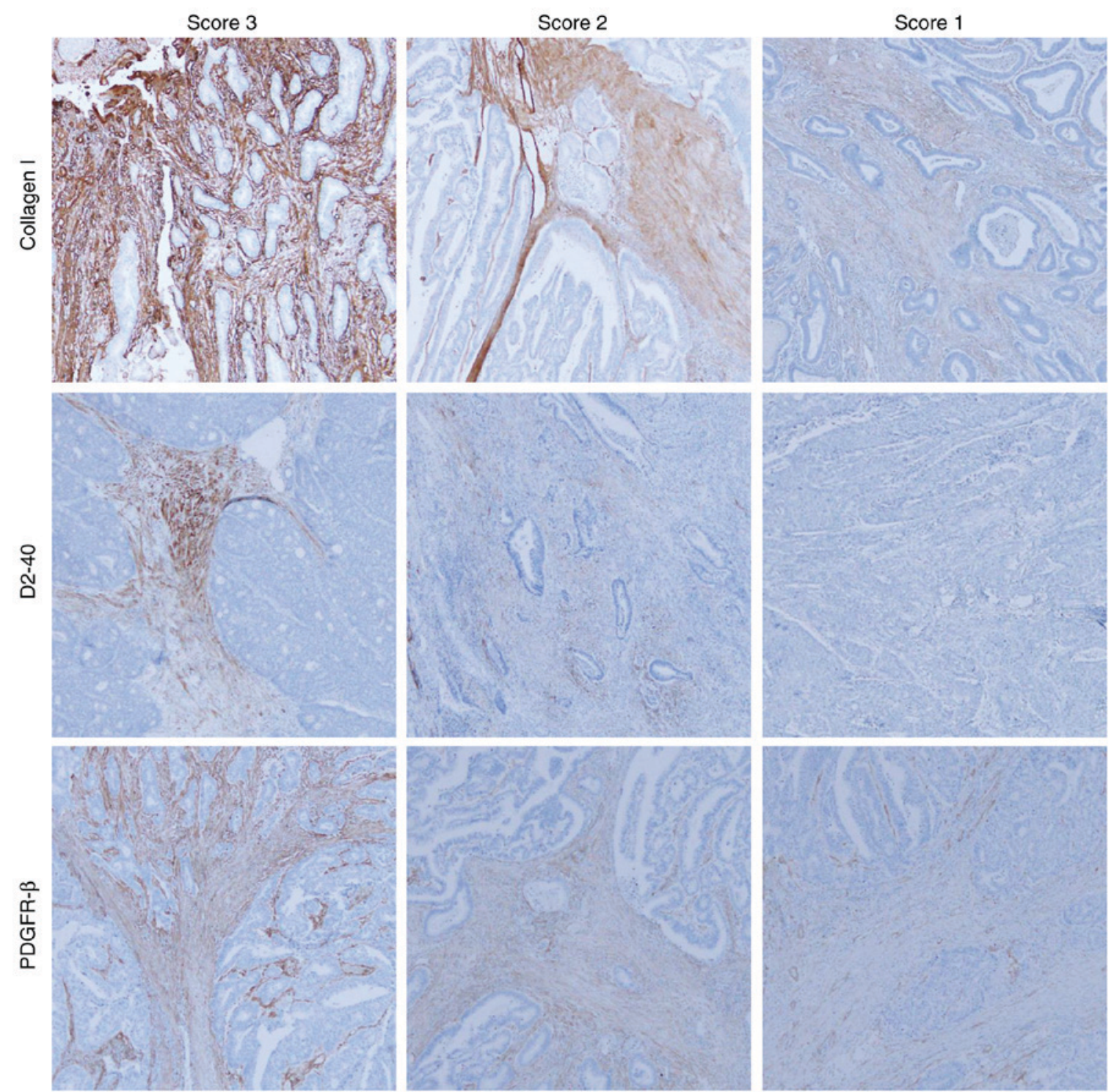

Figure 2. Intensity score for collagen I, D2-40, and platelet-derived growth factor receptor- $\beta$ (PDGFR- $\beta$; magnification, $x 40$ ). The expression intensity was evaluated by scoring in collagen I, D2-40, and PDGFR- $\beta$. Score 1, low intensity; score 2, moderate intensity; and score 3, high intensity. 
Table I. Clinicopathological characteristics of 121 colorectal cancer cases.

\begin{tabular}{lc}
\hline Clinicopathological features & Number of patients (\%) \\
\hline Age, median (range) & $67.4(26-93)$ \\
Sex & \\
Male & $66(54.5)$ \\
Female & $55(45.5)$ \\
Location & \\
Colon & $77(63.6)$ \\
Rectum & $44(36.4)$ \\
Histological type & \\
Well-differentiated tubular & $11(9.1)$ \\
adenocarcinoma (tub1) & \\
Moderately differentiated tubular & $99(81.8)$ \\
adenocarcinoma (tub2) & $3(2.5)$ \\
Papillary adenocarcinoma (pap) & $5(4.1)$ \\
Poorly differentiated & \\
adenocarcinoma (por) & $3(2.5)$ \\
Mucinous adenocarcinoma (muc) & \\
Stage & $64(52.9)$ \\
IIA & $48(39.7)$ \\
IIIB & $9(7.4)$ \\
IIIC & \\
Venous invasion & $89(73.6)$ \\
Low (v0, v1) & $32(26.4)$ \\
High (v2, v3) & $80(66.2)$ \\
Lymphatic invasion & $41(33.8)$ \\
Low (ly0, ly1) & \\
High (ly2, ly3) & $64(52.9)$ \\
Lymph node metastasis & $57(47.1)$ \\
Negative & \\
Positive & \\
\hline
\end{tabular}

cancer cases are summarized in Table I. We analyzed immunohistochemical expression area (ratio; percentage) of colorectal stroma. Mean/median expression areas of CAF/vessel markers were $26.787 \%$ (collagen I, range $0.848-59.069 \%$ ), $1.372 \%$ (D2-40, range 0.002-11.860\%), $11.646 \%$ (PDGFR- $\beta$, range $0.014-38.381 \%$ ), $15.372 \%$ ( $\alpha$-SMA subtraction, range $1.552-38.608 \%), 3.635 \%$ (CD31, range $0.249-11.071 \%$ ), and $2.226 \%$ (CD34, range $0.681-8.508 \%$ ) in 121 colorectal cancer specimens. There was no statistical association between any CAF/vessel markers (both of expression area/intensity) and the histological type (Table II).

Expression of CAF/vessel markers, and venous invasion/lymphatic invasion/lymph node metastasis. Relationships between the expression of CAF/vessel markers, and venous invasion/lymphatic invasion/lymph node metastasis are summarized in Tables III, IV and V, respectively. Extensive expression area of $\alpha$-SMA subtraction $(\mathrm{P}=0.002)$, collagen I $(\mathrm{P}=0.040)$ and PDGFR- $\beta(\mathrm{P}=0.040)$ were significantly correlated with high-grade venous invasion (Table III).
There was no relation between lymphatic invasion and expression of CAF/vessel markers except for CD34. Much expression area of CD34 was correlated with high lymphatic invasion $(\mathrm{P}=0.048)$ (Table IV). There was no relation between lymph node metastasis and expression of CAF/vessel markers except for $\alpha$-SMA subtraction (Table $V$ ). Weak expression area of $\alpha$-SMA subtraction was significantly correlated with positive lymph node metastasis $(\mathrm{P}=0.025)$. Strong expression area of $\alpha$-SMA subtraction was significantly correlated with negative lymph node metastasis $(\mathrm{P}=0.025)$.

Correlation among CAF/vessel markers. We evaluated the correlation among the expression of $\mathrm{CAF} /$ vessel markers. Speaman's correlation rho, and the scatter plots are shown in Table VI, and Fig. 3, respectively. There was significant correlation between $\alpha$-SMA subtraction and collagen $\mathrm{I}(\mathrm{P}<0.001$, correlation rho $=0.509)$ in expression area. The significant difference was not found between other CAF and vascular marker nor other CAF markers each other in expression area. There was not significant difference between collagen I and D2-40 ( $\mathrm{P}=0.119)$, collagen I and PDGFR- $\beta(\mathrm{P}=0.665)$, PDGFR- $\beta$ and D2-40 ( $\mathrm{P}=0.940)$ in intensity score. There was not significant difference between $\alpha$-SMA subtraction (expression area) and three each CAF markers intensity score; collagen I ( $\mathrm{P}=0.072), \mathrm{D} 2-40(\mathrm{P}=0.297)$, and PDGFR- $\beta$ $(\mathrm{P}=0.386)$. There was not difference between CD31 expression area and three CAF markers of intensity score; collagen I $(\mathrm{P}=0.232), \mathrm{D} 2-40(\mathrm{P}=0.205)$, and PDGFR- $\beta(\mathrm{P}=0.657)$. There was not difference between CD34 expression area and three CAF markers of intensity score; collagen I $(\mathrm{P}=0.133), \mathrm{D} 2-40$ $(\mathrm{P}=0.090)$, and PDGFR- $\beta(\mathrm{P}=0.641)$.

\section{Discussion}

Previous reports were accomplished about each CAF $(13,16-19)$, but did not provided the information about the comparison of each CAF. We captured immunohistochemical staining images, and adjusted phases in each case by using Adobe Photoshop software (Adobe ${ }^{\circledR}$ Photoshop $^{\circledR}$ CC $2014^{\circledR}$; USA) for image registration, and compared the different antibodies in the same field. In the present study, we analyzed histopathological expression of CAF markers (collagen I, D2-40, PDGFR- $\beta$ and $\alpha$-SMA subtraction) in 121 cases of the surgically resected advanced colorectal cancers, using digital image analyses. High levels of $\alpha$-SMA subtraction $(\mathrm{P}=0.002)$, collagen $\mathrm{I}(\mathrm{P}=0.040)$, and PDGFR- $\beta$ $(\mathrm{P}=0.040)$ expression areas tended to be associated with high venous invasion. $\alpha$-SMA positive and desmin negative myofibroblasts in the advanced colorectal cancer is associated with malignant potential in previous study $(16,17)$. Serum levels of Collagen I degradation telopeptide are correlated with staging and poor disease-free survival of colorectal patients (19). PDGFR- $\beta$ expression in colorectal cancer stroma is associated with metastatic potential (18). Our data supported these previous reports. $\alpha$-SMA subtraction and venous invasion had strongest positive correlation in the three markers ( $\alpha$-SMA subtraction, collagen I, and PDGFR- $\beta$ ), in spite of a median expression of the $\alpha$-SMA subtraction not being so high ( $\alpha$-SMA subtraction $15.372 \%$, collagen I 26.787\%, and PDGFR- $\beta$ 11.646\%). 
Table II. Expression of CAFs/vessel markers and histological type of colorectal cancer.

\begin{tabular}{|c|c|c|c|}
\hline Histological type & $\begin{array}{c}\text { Differentiated type } \\
\text { (tub1, tub2, pap) } n=113(93.4 \%)\end{array}$ & $\begin{array}{l}\text { Poorly differentiated type } \\
\text { (por, muc) } n=8(6.6 \%)\end{array}$ & P-value \\
\hline \multicolumn{4}{|l|}{ Expression area } \\
\hline Collagen I & 27.020 & 23.494 & 0.361 \\
\hline D2-40 & 1.372 & 1.178 & 0.449 \\
\hline PDGFR- $\beta$ & 11.759 & 8.614 & 0.249 \\
\hline$\alpha$-SMA subtraction & 15.372 & 15.034 & 0.718 \\
\hline CD31 & 3.635 & 3.620 & 0.726 \\
\hline CD34 & 2.226 & 2.343 & 0.888 \\
\hline \multicolumn{4}{|l|}{ Expression intensity } \\
\hline Collagen I & & & 0.719 \\
\hline High & $58(47.9)$ & $5(4.1)$ & \\
\hline Low & $55(45.5)$ & $3(2.5)$ & \\
\hline D2-40 & & & 0.481 \\
\hline High & $45(37.2)$ & $2(1.7)$ & \\
\hline Low & $68(56.2)$ & $6(5.0)$ & \\
\hline PDGFR- $\beta$ & & & 0.481 \\
\hline High & $45(37.2)$ & $2(1.7)$ & \\
\hline Low & $68(56.2)$ & $6(5.0)$ & \\
\hline
\end{tabular}

CAF, cancer-associated fibroblast. Differentiated type: Well-differentiated tubular adenocarcinoma (tub1), moderately differentiated tubular adenocarcinoma (tub2), and papillary adenocarcinoma (pap). Poorly differentiated type: Poorly differentiated adenocarcinoma (por) and mucinous adenocarcinoma (muc). PDGFR- $\beta$, platelet-derived growth factor- $\beta ; \alpha$-SMA, $\alpha$-smooth muscle actin.

Table III. Expression of CAF/vessel markers and venous invasion of colorectal cancer.

\begin{tabular}{|c|c|c|c|}
\hline Venous invasion & $\begin{array}{l}\text { Low }(v 0, v 1) \\
n=89(73.6 \%)\end{array}$ & $\begin{array}{l}\text { High (v2, v3) } \\
n=32(26.4 \%)\end{array}$ & $\mathrm{P}$-value \\
\hline \multicolumn{4}{|l|}{ Expression area } \\
\hline Collagen I & 25.615 & 30.048 & $0.040^{\mathrm{a}}$ \\
\hline D2-40 & 2.339 & 2.338 & 0.971 \\
\hline PDGFR- $\beta$ & 12.590 & 16.835 & $0.040^{\mathrm{a}}$ \\
\hline$\alpha$-SMA subtraction & 14.820 & 19.377 & $0.002^{\mathrm{a}}$ \\
\hline CD31 & 4.271 & 4.098 & 0.570 \\
\hline CD34 & 2.516 & 2.391 & 0.993 \\
\hline \multicolumn{4}{|l|}{ Expression intensity } \\
\hline Collagen I & & & 0.168 \\
\hline High & $43(35.5)$ & $20(16.5)$ & \\
\hline Low & $46(38.0)$ & $12(9.9)$ & \\
\hline D2-40 & & & 0.277 \\
\hline High & $32(26.4)$ & $15(12.4)$ & \\
\hline Low & $57(47.1)$ & $17(14.0)$ & \\
\hline PDGFR- $\beta$ & & & 0.506 \\
\hline High & $33(27.3)$ & $14(11.6)$ & \\
\hline Low & $56(46.3)$ & $18(14.9)$ & \\
\hline
\end{tabular}

${ }^{\mathrm{a}} \mathrm{P}<0.05$, statistical significance CAF, cancer-associated fibroblast; PDGFR- $\beta$, platelet-derived growth factor- $\beta ; \alpha$-SMA, $\alpha$-smooth muscle actin.

We evaluated the correlation among the expression of $\mathrm{CAF} /$ vessel markers. Collagen I, $\alpha$-SMA subtraction, and
PDGFR- $\beta$ correlated with venous invasion. There was significant correlation between $\alpha$-SMA subtraction and collagen I 
Table IV. Expression of CAF/vessel markers and lymphatic invasion of colorectal cancer.

\begin{tabular}{|c|c|c|c|}
\hline Lymphatic invasion & Low (ly0, ly1) n=80 (66.1\%) & High (ly2, ly3) n=41 (33.9\%) & P-value \\
\hline \multicolumn{4}{|l|}{ Expression area } \\
\hline Collagen I & 26.333 & 27.673 & 0.509 \\
\hline D2-40 & 2.026 & 2.950 & 0.156 \\
\hline PDGFR- $\beta$ & 12.789 & 15.515 & 0.188 \\
\hline$\alpha$-SMA subtraction & 15.287 & 17.465 & 0.119 \\
\hline CD31 & 4.113 & 4.443 & 0.665 \\
\hline CD34 & 2.301 & 2.838 & $0.048^{\mathrm{a}}$ \\
\hline \multicolumn{4}{|l|}{ Expression intensity } \\
\hline Collagen I & & & 0.604 \\
\hline High & $43(35.5)$ & $20(16.5)$ & \\
\hline Low & $37(30.6)$ & $21(17.4)$ & \\
\hline D2-40 & & & 0.225 \\
\hline High & $28(23.1)$ & $19(15.7)$ & \\
\hline Low & $52(43.0)$ & $22(18.2)$ & \\
\hline PDGFR- $\beta$ & & & 0.715 \\
\hline High & $32(26.4)$ & $15(12.4)$ & \\
\hline Low & $48(39.7)$ & $26(21.5)$ & \\
\hline
\end{tabular}

${ }^{\mathrm{a}} \mathrm{P}<0.05$, statistical significance CAF, cancer-associated fibroblast; PDGFR- $\beta$, platelet-derived growth factor receptor- $\beta ; \alpha$-SMA, $\alpha$-smooth muscle actin.

Table V. Expression of CAF/vessel markers and lymph node metastasis of colorectal cancer.

\begin{tabular}{|c|c|c|c|}
\hline Lymph node metastasis & Negative $n=64(52.9 \%)$ & Positive $n=57(47.1 \%)$ & P-value \\
\hline \multicolumn{4}{|l|}{ Expression area } \\
\hline Collagen I & 26.798 & 26.775 & 0.990 \\
\hline D2-40 & 2.228 & 2.463 & 0.698 \\
\hline PDGFR- $\beta$ & 13.943 & 13.454 & 0.882 \\
\hline$\alpha$-SMA subtraction & 17.242 & 14.659 & $0.025^{\mathrm{a}}$ \\
\hline CD31 & 3.627 & 4.098 & 0.704 \\
\hline CD34 & 2.350 & 2.632 & 0.352 \\
\hline \multicolumn{4}{|l|}{ Expression intensity } \\
\hline Collagen I & & & 0.168 \\
\hline High & $33(27.3)$ & $30(24.8)$ & \\
\hline Low & $31(25.6)$ & $27(22.3)$ & \\
\hline D2-40 & & & 0.277 \\
\hline High & $23(19.0)$ & $24(19.8)$ & \\
\hline Low & $41(33.9)$ & $33(27.3)$ & \\
\hline PDGFR- $\beta$ & & & 0.506 \\
\hline High & $24(19.8)$ & $23(19.0)$ & \\
\hline Low & $40(33.1)$ & $34(28.1)$ & \\
\hline
\end{tabular}

${ }^{\mathrm{a}} \mathrm{P}<0.05$, statistical significance CAF, cancer-associated fibroblast; PDGFR- $\beta$, platelet-derived growth factor receptor- $\beta$; $\alpha$-SMA, $\alpha$-smooth muscle actin.

expression $(\mathrm{P}<0.001$, correlation $\mathrm{rho}=0.509)$. $\mathrm{PDGFR}-\beta$ was not associated with collagen I nor $\alpha$-SMA subtraction image, though high PDGFR- $\beta$ expression was correlated with venous invasion. These data suggested that $\alpha$-SMA subtraction, collagen I, and PDGFR- $\beta$ might have differential strength effects for venous invasion and different expression patterns in advanced colorectal cancer stroma. Immunohistochemically, collagen I, $\alpha$-SMA subtraction, and PDGFR- $\beta$ widely 
Table VI. Spearman's rank correlation rho in CAFs/vessel markers.

\begin{tabular}{|c|c|c|c|c|c|c|}
\hline & Collagen I & D2-40 & PDGFR- $\beta$ & $\alpha$-SMA subtraction & CD31 & CD34 \\
\hline Collagen I & & 0.150 & 0.260 & $0.509^{\mathrm{a}}$ & 0.088 & 0.133 \\
\hline D2-40 & & & 0.145 & 0.257 & 0.227 & 0.237 \\
\hline PDGFR- $\beta$ & & & & 0.318 & 0.127 & 0.086 \\
\hline$\alpha$-SMA subtraction & & & & & 0.060 & 0.145 \\
\hline CD31 & & & & & & 0.319 \\
\hline CD34 & & & & & & \\
\hline
\end{tabular}

${ }^{a} \mathrm{P}>0$.4 . CAF, cancer-associated fibroblast; PDGFR- $\beta$, platelet-derived growth factor receptor- $\beta ; \alpha$-SMA, $\alpha$-smooth muscle actin.

A (\%)

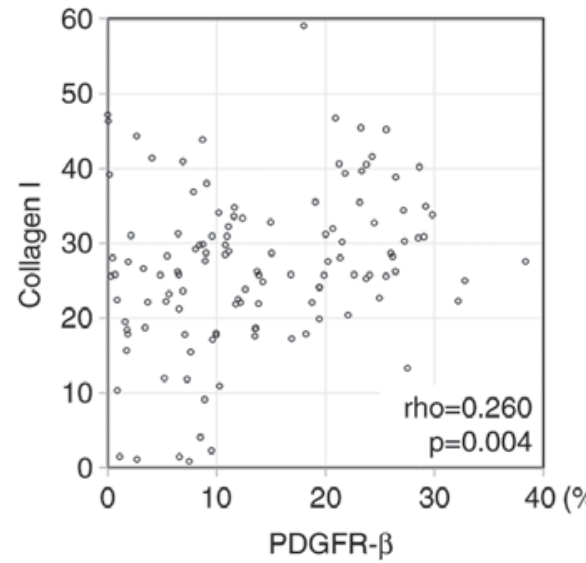

B (\%)

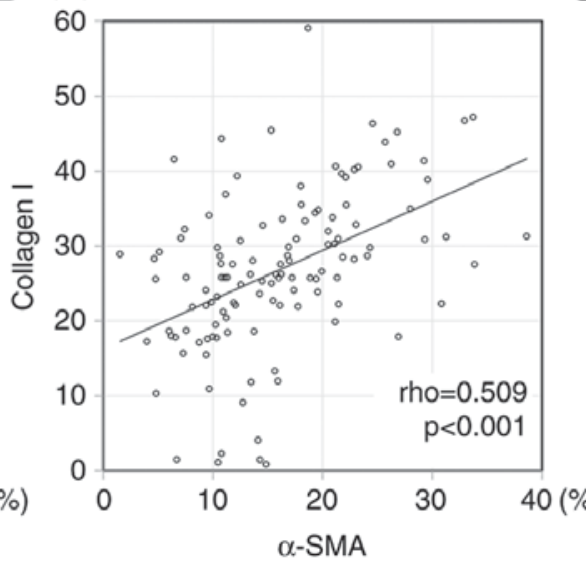

C (\%)

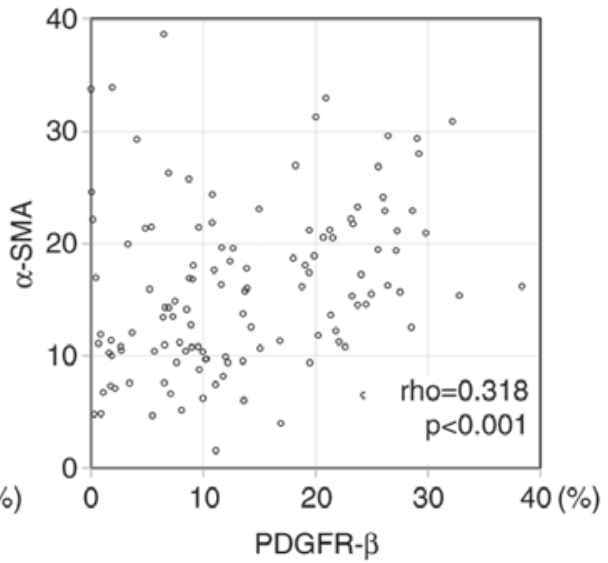

Figure 3. The Scatter plot for expression of platelet-derived growth factor receptor- $\beta$ (PDGFR- $\beta$ ) and collagen I. (A) $\alpha-$ SMA subtraction and collagen I, (B) and PDGFR- $\beta$ and (C) $\alpha$-SMA subtraction in 121 colorectal cancer stromas. There was significant correlation between expression of $\alpha$-SMA subtraction and collagen I ( $\mathrm{r}=0.509)$. There were no correlations between PDGFR- $\beta$ and collagen $\mathrm{I}(\mathrm{r}=0.260)$ nor PDGFR- $\beta$ and $\alpha-\mathrm{SMA}$ subtraction $(\mathrm{r}=0.318)$.

expressed in the whole colorectal cancer stroma. On the other hand, the expression of D2-40 was generally localized in the cancer stroma, and was more frequently detected in the superficial parts of the cancer tissue. The expression patterns (location, intensity) of collagen I were similar to that of $\alpha$-SMA subtraction image, but PDGFR- $\beta$ and D2-40 showed different expression patterns in advanced colorectal cancer stroma. The different expression pattern may influence venous invasion. It is necessary to further study the relationships between CAFs in colorectal cancer. CAF markers must become potential targets of future colorectal cancer treatment. Combination therapy of PDGFR tyrosine kinase inhibitor and anticancer drug was more effective than the anticancer drug alone $(20,21)$. In the future, cancer treatment will be taylor made treatment, and its options will be expanded. Knowing the detailed characters of CAFs leads to taylor made treatment. In our study, it is possible that $\alpha$-SMA subtraction contributes most to venous invasion compare with collagen I, PDGFR- $\beta$.

The significant differences were seen in the expression of collagen I, PDGFR- $\beta$ and $\alpha$-SMA subtraction between low and high venous invasion. Expression area analysis was quantifiable by using digital image analyses. On the other hand, it was difficult to analyze the intensity of collagen I, and $\alpha$-SMA subtraction, because the expression of $\alpha$-SMA subtraction and collagen I was highly expressed in most cases.
The intensity of the expression of PDGFR- $\beta$ is likely to be unstable due to its small fluctuation in intensity. Therefore, there was no significant difference in the expression intensity. Interestingly, the expression of $\alpha$-SMA subtraction was low in lymph node metastasis cases in this study. We analyzed the CAF and vessel markers focused on the D2-40 expressed lesions, therefore D2-40 might have some influence for CAF and vessel markers expression. D2-40 (Podoplanin) is a $38-\mathrm{kDa}$ mucin-type transmembrane glycoprotein with extensive $\mathrm{O}$-glycosylation and high sialic acid content, and it has been implicated in tumor progression (22). Podoplanin promotes relocalization of ezrin to filopodia-like structure and platelet aggregation, so that podoplanin may be involved in cancer migration, invasion, and malignant progression (23-25). The majority of recently reports identified D2-40 expression of CAFs as an unfavorable marker of prognosis (9-11), but some reports described D2-40 in CAFs as a favorable marker for colorectal cancer $(12,13)$. Choi et al analyzed early and advanced colorectal cancer (12). Yamanashi et al analyzed 120 advanced colorectal cancer cases (13). The detailed mechanism has not been understood why the expression of D2-40 becomes a favorable marker for colorectal cancer. Despite high venous invasion of $\alpha$-SMA subtraction expression, D2-40 expressed area might influence the potential of lymph node metastasis in this study. Collagen 
I and PDGFR- $\beta$ are recognized to have malignant potential in CAFs of colorectal cancer $(18,19)$. These two markers do not have a relationship with lymph node metastases, but have a relationship with vein involvement in our study. There is possibility that $\alpha$-SMA subtraction, collagen I, and PDGFR- $\beta$ are associated with venous invasion rather than lymphatic invasion. Both vessel markers (CD31/CD34) were not statistically associated with CAF markers/other histological factors, except for the relationship between CD34 and lymphatic invasion.

Our results indicated that the patterns of expression for $\alpha$-SMA subtraction, collagen I, D2-40, and PDGFR- $\beta$ vary in CAFs of advanced colorectal cancer. Collagen I, $\alpha$-SMA subtraction, and PDGFR- $\beta$ were widely distributed in the colorectal cancer stroma, while D2-40 was limited. The expression of $\alpha$-SMA subtraction, collagen I, and PDGFR- $\beta$ were associated with high venous invasion. However, the relationship between CAF markers might be complicated to understand. There have been any previous studies for the relationships between CAF markers. We must further study CAF markers by analyzing with variable viewpoints (i.e., expression pattern, relationship between CAFs and strength for clinicopathological factors).

\section{Acknowledgements}

This study was supported by Grants-in Aid for Science from the Ministry of Education, Culture, Sports, Science and Technology in Japan and a grant for Hirosaki University Institutional Research.

\section{References}

1. Orimo A, Gupta PB, Sgroi DC, Arenzana-Seisdedos F, Delaunay T, Naeem R, Carey VJ, Richardson AL and Weinberg RA: Stromal fibroblasts present in invasive human breast carcinomas promote tumor growth and angiogenesis through elevated SDF-1/CXCL12 secretion. Cell 121: 335-348, 2005.

2. Erez N, Truitt M, Olson P, Arron ST and Hanahan D: Cancer-associated fibroblasts are activated in incipient neoplasia to orchestrate tumor-promoting inflammation in an NF-kappaB-dependent manner. Cancer Cell 17: 135-147, 2010.

3. Olumi AF, Grossfeld GD, Hayward SW, Carroll PR, Tlsty TD and Cunha GR: Carcinoma-associated fibroblasts direct tumor progression of initiated human prostatic epithelium. Cancer Res 59: 5002-5011, 1999.

4. Yang G, Rosen DG, Zhang Z, Bast RC Jr, Mills GB, Colacino JA, Mercado-Uribe I and Liu J: The chemokine growth-regulated oncogene 1 (Gro-1) links RAS signaling to the senescence of stromal fibroblasts and ovarian tumorigenesis. Proc Natl Acad Sci USA 103: 16472-16477, 2006.

5. Hwang RF, Moore T, Arumugam T, Ramachandran V, Amos KD, Rivera A, Ji B, Evans DB and Logsdon CD: Cancer-associated stromal fibroblasts promote pancreatic tumor progression. Cancer Res 68: 918-926, 2008.

6. Hu M, Peluffo G, Chen H, Gelman R, Schnitt S and Polyak K: Role of COX-2 in epithelial-stromal cell interactions and progression of ductal carcinoma in situ of the breast. Proc Natl Acad Sci USA 106: 3372-3377, 2009.

7. Togo S, Polanska UM, Horimoto $Y$ and Orimo A: Carcinoma-associated fibroblasts are a promising therapeutic target. Cancers (Basel) 5: 149-169, 2013.

8. Pula B, Witkiewicz W, Dziegiel P and Podhorska-Okolow M: Significance of podoplanin expression in cancer-associated fibroblasts: A comprehensive review. Int J Oncol 42: 1849-1857, 2013.
9. Kitano H, Kageyama S, Hewitt SM, Hayashi R, Doki Y, Ozaki Y, Fujino S, Takikita M, Kubo H and Fukuoka J: Podoplanin expression in cancerous stroma induces lymphangiogenesis and predicts lymphatic spread and patient survival. Arch Pathol Lab Med 134: 1520-1527, 2010.

10. Pula B, Jethon A, Piotrowska A, Gomulkiewicz A, Owczarek T, Calik J, Wojnar A, Witkiewicz W, Rys J, Ugorski M, et al: Podoplanin expression by cancer-associated fibroblasts predicts poor outcome in invasive ductal breast carcinoma. Histopathology 59: 1249-1260, 2011.

11. Schoppmann SF, Jesch B, Riegler MF, Maroske F, Schwameis K, Jomrich $\mathrm{G}$ and Birner P: Podoplanin expressing cancer associated fibroblasts are associated with unfavourable prognosis in adenocarcinoma of the esophagus. Clin Exp Metastasis 30: 441-446, 2013.

12. Choi SY, Sung R, Lee SJ, Lee TG, Kim N, Yoon SM, Lee EJ, Chae HB, Youn SJ and Park SM: Podoplanin, $\alpha$-smooth muscle actin or S100A4 expressing cancer-associated fibroblasts are associated with different prognosis in colorectal cancers. J Korean Med Sci 28: 1293-1301, 2013.

13. Yamanashi T, Nakanishi Y, Fujii G, Akishima-Fukasawa Y, Moriya Y, Kanai Y, Watanabe M and Hirohashi S: Podoplanin expression identified in stromal fibroblasts as a favorable prognostic marker in patients with colorectal carcinoma. Oncology 77: 53-62, 2009.

14. InternationalUnion AgainstCancer:TNMClassification of Malignant Tumours. Sobin LH, Gospodarowicz MK and Wittekind CH (eds): 7th Edition. Wiley-Blackwell, New York, NY, 2009.

15. Japanese Society for Cancer of the Colon and Rectum: Japanese Classification of Colorectal Carcinoma. Kanehara \& Co., Ltd., Tokyo, 2013.

16. Takatsuna M, Morohashi S, Yoshizawa T, Hirai H, Haga T, Ota R, Saito K, Wu Y, Seino H, Aoyagi Y, et al: Myofibroblast distribution is associated with invasive growth types of colorectal cancer. Oncol Rep 36: 3154-3160, 2016.

17. Takatsuna M, Morohashi S, Yoshizawa T, Hirai H, Haga T, Ota R, Wu Y, Morohashi H, Hakamada K, Terai S and Kijima H: Myofibroblasts of the muscle layer stimulate the malignant potential of colorectal cancer. Oncol Rep 36: 1251-1257, 2016.

18. Kitadai Y,Sasaki T, Kuwai T,Nakamura T, Bucana CD, Hamilton SR and Fidler IJ: Expression of activated platelet-derived growth factor receptor in stromal cells of human colon carcinomas is associated with metastatic potential. Int J Cancer 119: 2567-2574, 2006.

19. Zou X, Feng B, Dong T, Yan G, Tan B, Shen H, Huang A, Zhang X, Zhang M, Yang P, et al: Up-regulation of type I collagen during tumorigenesis of colorectal cancer revealed by quantitative proteomic analysis. J Proteomics 94: 473-485, 2013.

20. Kitadai Y, Sasaki T, Kuwai T, Nakamura T, Bucana CD and Fidler IJ: Targeting the expression of platelet-derived growth factor receptor by reactive stroma inhibits growth and metastasis of human colon carcinoma. Am J Pathol 169: 2054-2065, 2006.

21. Sumida T, Kitadai Y, Shinagawa K, Tanaka M, Kodama M, Ohnishi M, Ohara E, Tanaka S, Yasui W and Chayama K: Anti-stromal therapy with imatinib inhibits growth and metastasis of gastric carcinoma in an orthotopic nude mouse model. Int $\mathrm{J}$ Cancer 128: 2050-2062, 2011.

22. Kono T, Shimoda M, Takahashi M, Matsumoto K, Yoshimoto T, Mizutani M, Tabata C, Okoshi K, Wada $\mathrm{H}$ and Kubo $\mathrm{H}$ : Immunohistochemical detection of the lymphatic marker podoplanin in diverse types of human cancer cells using a novel antibody. Int J Oncol 31: 501-508, 2007.

23. Wicki A, Lehembre F, Wick N, Hantusch B, Kerjaschki D and Christofori G: Tumor invasion in the absence of epithelial-mesenchymal transition: Podoplanin-mediated remodeling of the actin cytoskeleton. Cancer Cell 9: 261-272, 2006.

24. Sugimoto $Y$, Watanabe $M$, Oh-hara $T$, Sato $S$, Isoe $T$ and Tsuruo T: Suppression of experimental lung colonization of a metastatic variant of murine colon adenocarcinoma 26 by a monoclonal antibody 8 F11 inhibiting tumor cell-induced platelet aggregation. Cancer Res 51: 921-925, 1991.

25. Watanabe M, Okochi E, Sugimoto Y and Tsuruo T: Identification of a platelet-aggregating factor of murine colon adenocarcinoma 26: $\mathrm{Mr} 44,000$ membrane protein as determined by monoclonal antibodies. Cancer Res 48: 6411-6416, 1988.

This work is licensed under a Creative Commons Attribution-NonCommercial-NoDerivatives 4.0 International (CC BY-NC-ND 4.0) License. 